\title{
Residual strain measurement of SOI wafer using convergent beam electron diffraction
}

\author{
K. Kuramochi, * T. Yamazaki, * T. Isaka, ${ }^{*}$ K. Watanabe, ${ }^{* *}$ and I. Hashimoto* \\ * Department of Physics, Tokyo University of Science, Tokyo, 162-8601, Japan \\ ** Tokyo Metropolitan College of Technology, Tokyo, 140-0011, Japan
}

Only technique that can provide information on the lattice strain with a higher spatial resolution and high accuracy is the convergent-beam electron diffraction (CBED), which uses higher-order Laue zone (HOLZ) lines in a bright field disk. The probes with a diameter of a few nanometers can easily be formed, thus it is well adapted to the dimensions in which the strain may vary. Consequently, there are a growing number of studies in which the technique has successfully been applied to many materials [1-3]. However, a lack of the accurate refinement algorithm has prevented the implementation of the CBED method as a standard technique for strain measurement, although some optimized algorithms based on the $\chi^{2}$-based minimization routine have already proposed. In this paper, we report the analytical technique combined with a detecting system of HOLZ lines based on both tracing the HOLZ lines by the Radon transform and background subtracting in energy unfiltered $\mathrm{CBED}$ for high precision measurements of local residual strain at a $\mathrm{SiO}_{2} / \mathrm{Si}$ interface in silicon on insulator (SOI) wafer.

The SOI wafer used in this paper has a sandwich structure of a $50 \mu \mathrm{m}$ thick $n$-type $\mathrm{Si}(100)$ layer with a resistivity of $10 \Omega \cdot \mathrm{cm}$, a $500 \mathrm{~nm}$ thick buried oxide (BOX) layer and an $n$-type $\operatorname{Si}(100)$ substrate. The [012] direction was chosen within a range of specimen tilt of a conventional TEM, because of the sufficient number of intense HOLZ lines and non-dispersive character of zeroth-layer dispersion surface.

In recent year the Hough transform and the Radon transform have been used to automatically extract straight HOLZ lines, where a discrete Radon transform corresponds to a Hough transform. The main advantages of the Radon transform are that it can easily be automated and makes use of the whole intensity distribution along a HOLZ line $[4,5]$. As examples, the raw CBED patterns observed at 1.0, 2.0 and $5.0 \mu \mathrm{m}$ distance from interface between BOX and Si substrate are displayed in Figs. 1a-c. Although the raw CBED patterns are increasingly obscure by closing to interface, strong first order Laue zone (FOLZ) lines are observed. In Figs. 1d-f, the FOLZ lines traced by the present detection system are superimposed on the image subtracting the background intensity. The central parts enclosed by the white squares in Figs. 1d-f are shown in Figs. 1g-i, respectively, because of ease of seeing. All traced HOLZ lines are on the maximum of each HOLZ line.

The fitting procedures between the experimental CBED pattern and kinematical simulated CBED pattern have been suggested by many researchers. Most investigations have been carried out by an optimized algorithm like simplex method or a try-and-error method. However, it is doubtful whether the optimized result is true and hard to utilize these methods as a routine work. In order to solve these problems, we suggest a different method, in which all lattice parameters are given analytically. As an example, the lattice parameters of [012]-orientated Si are determined. A crystal coordinate is transformed as the $c$-axis set equal to the incident beam direction and glazing angles set equal to $90^{\circ}$. When the small strains, $\Delta a, \Delta b, \Delta c, \Delta \alpha, \Delta \beta$ and $\Delta \gamma$, are assumed in this system, the coordinates of the intersections between HOLZ lines are represented as simultaneous-linear equations using these lattice parameters if the second orders of small strains can be neglected. 
Therefore, all lattice parameters can be analytically calculated. It is noted that $\Delta \alpha$ and $\Delta \beta$ are neglected due to their small values compared with the other parameters in this coordinate.

The distribution of residual strain components as a function of the distance from the interfaces of $\mathrm{SiO}_{2} / \mathrm{Si}$ interface is obtained as shown in Fig. 2, where the vertical gray band expresses the position of BOX. The strain measured by micro Raman spectroscopy is also displayed by solid line. Although there are the large error bars at respective measurement, the distribution of residual strain is clearly shown. From both measurements, it is found that there is the slight tensile stress at the side of Si substrate, and the compressive stress at the side of Si overlay.

\section{References}

[1] Y. Tomokiyo, et al., Ultramicroscopy 54 (1994) 276.

[2] A. Toda, N. Ikarashi and H. Ono, J. Crystal Growth 210 (2000) 341.

[3] S. J. Rozeveld and J. M. Howe, Ultramicroscopy 50 (1993) 41.

[4] S. Krämer and J. Mayer, J. Microsc. 194 (1999) 2.

[5] P. V. C. Hough, US Patent 3069654 (1962).

[6] This research was financially supported by Kazato Research Foundation.
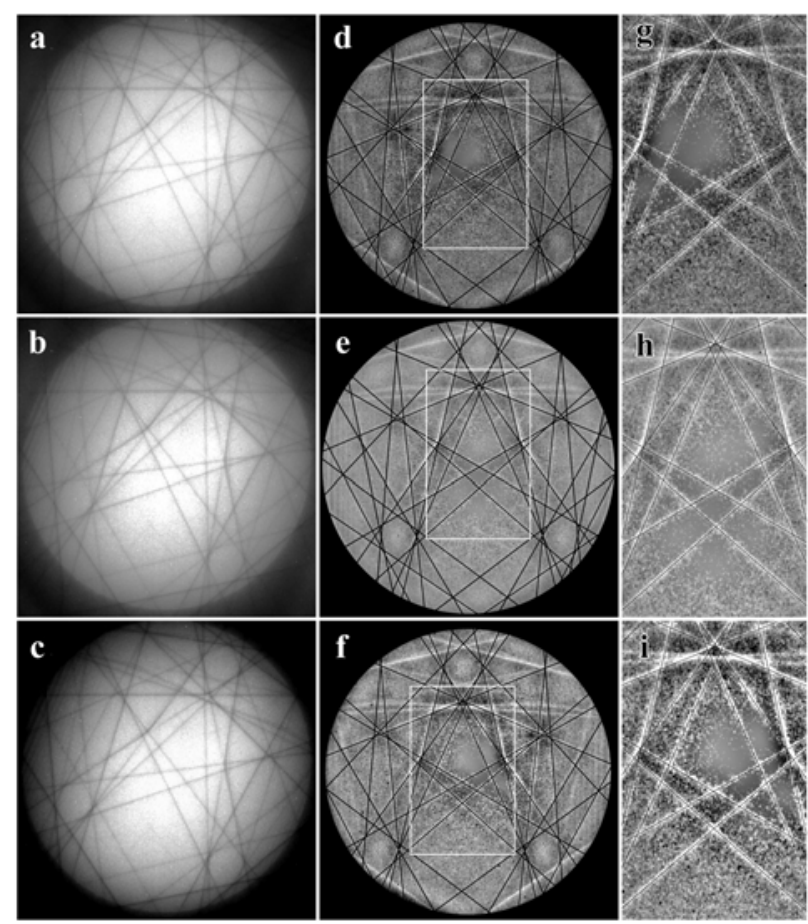

FIG. 1. (a)-(c) Raw experimental CBED pattern taken at 1.0, 2.0 and $5.0 \mu \mathrm{m}$ distance from the interface between BOX and $\mathrm{Si}$ substrate, (d)-(f) CBED patterns superimposed the traced HOLZ lines, and (g)(i) enlarged images at white square in Figs. 1(d)-(f).

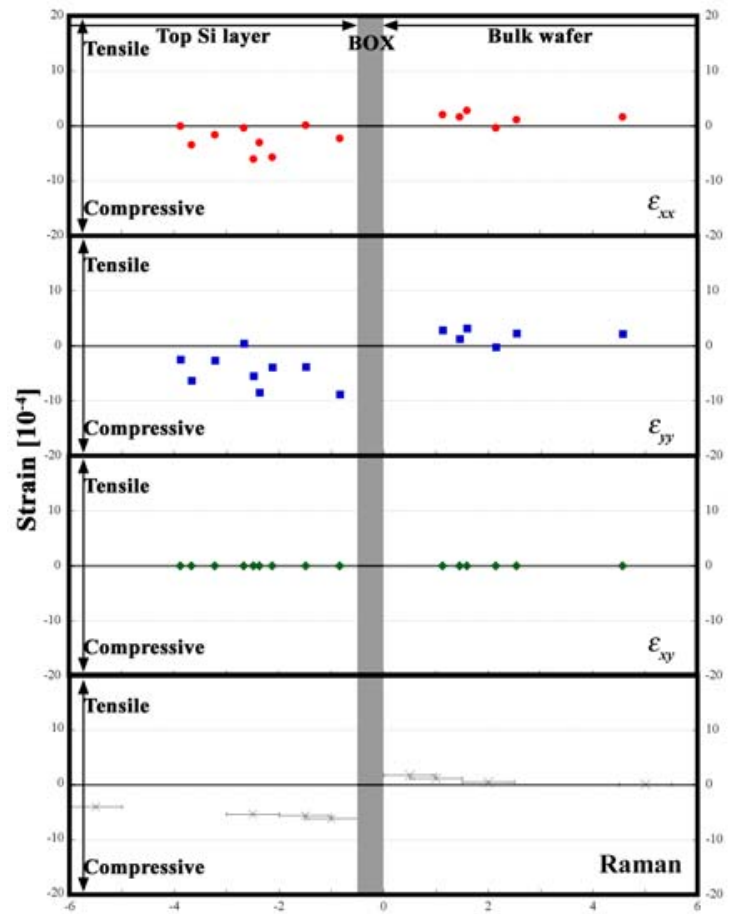

Distance from interace between BOX and bulk wafer $[\mu \mathrm{m}]$

FIG. 2. The residual stress measured by CBED and Raman spectroscopy. $\varepsilon_{x x}$ and $\varepsilon_{y y}$ are the stress component along [001] and [120] directions. 